\title{
Generalized Multilevel Physical Optics (MLPO) for Comprehensive Analysis of Reflector Antennas
}

\author{
Christine Letrou, Senior Member, IEEE, and Amir Boag, Fellow, IEEE
}

\begin{abstract}
Recent developments of the Multilevel Physical Optics (MLPO) algorithm aiming at the comprehensive analysis of complex reflector antenna systems are presented. The Physical Theory of Diffraction (PTD) line integral along the rim of a reflector is combined with the Physical Optics (PO) surface integral within the multilevel algorithm. The multilevel scheme is also generalized to combine fields radiated by various components of different sizes, as encountered in complex antenna systems with multiple feeds and/or reflectors. Comparison with published results demonstrates the ability of the MLPO algorithm to cope accurately and efficiently with realistic reflector antenna problems.
\end{abstract} Ministry of Research, France.

C. Letrou is with the Institut Telecom, Telecom SudParis, CNRS Lab. SAMOVAR, Evry, France (email: christine.letrou@it-sudparis.eu).

A. Boag is with the School of Electrical Engineering, Tel Aviv University, Tel Aviv 69978, Israel (e-mail: boag@eng.tau.ac.il). 


\section{Generalized Multilevel Physical Optics (MLPO) for Comprehensive Analysis of Reflector Antennas}

\section{INTRODUCTION}

$\mathbf{T}$ HE Physical Optics (PO) approximation provides an attractive computational tool for the analysis of large reflector antennas [1]. The PO combined with the PTD (e.g. in the form of Incremental Length Diffraction Coefficients [2], [3]) often strikes a balance between the computational burden and accuracy requirements for the reflector antenna analysis. The PO-PTD combination facilitates uniformly accurate evaluation of the co- and cross-polarized radiation patterns, including the far sidelobe regions.

Numerically rigorous techniques such as the method of moments, though more accurate, are considerably more computationally demanding and, therefore, mostly employed for small and moderately sized antennas. Combining or hybridizing a method of moments technique with a PO-type surface integration is often the preferred method to address reflector antenna problems involving evaluation of the current on the reflector surface [4]. In contrast, computationally inexpensive Geometrical Theory of Diffraction (GTD), often used to compute fields in far-out sidelobes, is subject to well-known limitations due to the presence of caustics [5], [6] and to the possibly large number of diffraction points in the case of shaped reflectors or reflectors with irregular edges. Also, GTD is valid only when edges are illuminated from the far field, which is not always the case in multiple reflector antennas. Hence GTD computations of cross-polarized patterns and far sidelobe levels are not considered accurate enough in the case of high performance reflector antennas [7]. A modified PO formulation recently proposed in [8] and [9] promises to describe the diffraction effects while performing only PO type surface integrals with normal vector directions modified based on the observation direction. This approach however is yet to be fully developed for arbitrary three-dimensional geometries.

The combined PO/PTD approach does not suffer from the above limitations, but for large reflectors and wide angle patterns, the straightforward evaluation of the pertinent integrals for a wide range of observation directions is inefficient due to its high computational complexity. This computational burden can pose a significant limitation in situations such as reflector shaping and optimization [10], as well as multibeam multifrequency systems, where repeated evaluation of antenna characteristics is required. The Fast Fourier Transform (FFT) facilitates numerically efficient evaluation of radiation integrals, but only for planar apertures. The MLPO was introduced in [11] in order to reduce the complexity of evaluating the PO integrals over arbitrary shaped surfaces to a level comparable to that of the FFT-based techniques. The efficacy of the MLPO approach for antenna analysis has already been demonstrated in the case of simple PO analysis (surface integrals only) of lens and reflector antennas.

In this paper, we show how the MLPO algorithm can be generalized for the efficient computation of wide angle radiation patterns, accommodating both diffraction and spill over effects. For the sake of simplicity, we present the new algorithm developments for the case of a single reflector antenna system. In Section II, we formulate the problem under study reducing it to the evaluation of PO and PTD integrals. The presentation of the generalized MLPO algorithm in Section III starts with an outline of the basic multilevel approach and proceeds with the computation of elemental sub-patterns including the PTD contribution followed by a hierarchical aggregation of reflector sub-patterns and additional contributions, such as the feed radiation, into the final pattern. A numerical example is worked out in Section IV to demonstrate the main features of the proposed approach.

\section{PROBLEM SPECIFICATION}

Consider a PO-based computation of the radiation pattern of an idealized reflector antenna comprising a primary feed and a single reflector surface. We define an antenna far field pattern $\boldsymbol{U}(\hat{\boldsymbol{r}})$ in direction $\hat{\boldsymbol{r}}$ as:

$$
\boldsymbol{U}(\hat{\boldsymbol{r}})=4 \pi r e^{j k r} \boldsymbol{E}(\boldsymbol{r}) \quad r \rightarrow \infty
$$

where $k$ is the wavenumber and $\boldsymbol{E}(\boldsymbol{r})$ is the far electric field radiated by the antenna at observation point $r=r \hat{r}$. In order to compute the reflector antenna wide angle pattern, the PO surface integral contributions have to be augmented with those of the PTD line integral along the reflector rim and further combined with the primary feed pattern. The resulting expression for the far field pattern radiated by the single reflector antenna system is then of the form:

$$
\begin{gathered}
\boldsymbol{U}(\hat{\boldsymbol{r}})=\int_{S} \boldsymbol{A}\left(\hat{\boldsymbol{r}}, \boldsymbol{r}^{s}\right) e^{j k \hat{\boldsymbol{r}} \cdot \boldsymbol{r}^{s}} d s+\int_{C} \boldsymbol{D}\left(\hat{\boldsymbol{r}}, \boldsymbol{r}^{c}\right) e^{j k \hat{\boldsymbol{r}} \cdot \boldsymbol{r}^{c}} d z_{l} \\
+\boldsymbol{U}_{\mathrm{f}}(\hat{\boldsymbol{r}}) e^{j k \hat{\boldsymbol{r}} \cdot \boldsymbol{r}^{\mathrm{f}}}
\end{gathered}
$$

where $S$ and $C$ denote the reflector surface and rim contour, respectively. The elemental surface contribution $\boldsymbol{A}\left(\hat{\boldsymbol{r}}, \boldsymbol{r}^{s}\right)$ is related to the equivalent currents on the surface and $\boldsymbol{D}\left(\hat{\boldsymbol{r}}, \boldsymbol{r}^{c}\right)$ stands for the Incremental Length Diffraction Coefficients (ILDCs). Also in (2), $\boldsymbol{U}_{\mathrm{f}}(\hat{\boldsymbol{r}})$ is the feed pattern in the feed centered coordinate system. It can be either known analytically or obtained via measurement, or from a separate numerical analysis of the feed system. Also, $\boldsymbol{r}^{s}$ denotes a point on the reflector surface $S, r^{c}$ a point on the reflector rim $C$, and $r^{\mathrm{f}}$ the position of the primary feed. These position vectors are defined in the same coordinate system, called the "observation" coordinate system. 
The fields over surface $S$ are assumed to be known thanks, e.g., to a known incident field and local impedance boundary conditions. In the following, we assume that $S$ is a perfect conductor. Then:

$$
\boldsymbol{A}\left(\hat{\boldsymbol{r}}, \boldsymbol{r}^{s}\right)=j 2 k \eta \hat{\boldsymbol{r}} \times\left[\hat{\boldsymbol{r}} \times\left(\hat{\boldsymbol{n}}\left(\boldsymbol{r}^{s}\right) \times \boldsymbol{H}^{\mathrm{f}}\left(\boldsymbol{r}^{s}\right)\right)\right]
$$

where $\eta=120 \pi$ is the intrinsic wave impedance, while $\boldsymbol{H}^{\mathrm{f}}\left(\boldsymbol{r}^{s}\right)$ and $\hat{\boldsymbol{n}}\left(\boldsymbol{r}^{s}\right)$ denote, respectively, the incident magnetic field produced by the feed and the outward unit vector normal to $S$, both at point $\boldsymbol{r}^{s}$ on the surface.

Along the rim, $\boldsymbol{D}\left(\hat{\boldsymbol{r}}, \boldsymbol{r}^{c}\right)$ is the far field pattern of the ILDCs given in [2], [3], normalized to the elemental length $d z_{l}$ :

$$
\begin{aligned}
& \boldsymbol{D}\left(\hat{\boldsymbol{r}}, \boldsymbol{r}^{c}\right)=\boldsymbol{D}^{(\mathrm{TM})}+\boldsymbol{D}^{(\mathrm{TE})} \\
& \text { with } \quad \boldsymbol{D}^{(\mathrm{TM})}=E_{z_{l}}^{\mathrm{f}} \frac{\sin \theta_{l}}{\sin ^{2} \theta_{0 l}} \frac{2 \sin \left(\phi_{0 l} / 2\right)}{\left|\cos \left(\phi_{0 l} / 2\right)\right|+\sin (\alpha / 2)} \hat{\boldsymbol{\theta}}_{l} \\
& \boldsymbol{D}^{(\mathrm{TE})}=-\operatorname{sgn}\left(\pi-\phi_{0 l}\right) \eta H_{z_{l}}^{\mathrm{f}} \frac{1}{\sin \theta_{0 l}} \\
& \times \frac{1}{\sin (\alpha / 2)\left(\left|\cos \left(\phi_{0 l} / 2\right)\right|+\sin (\alpha / 2)\right)} \\
& \times\left\{\sin \phi_{l} \hat{\boldsymbol{\phi}}_{l}-\left[\cos \phi_{l} \cos \theta_{l}+(1+2\right.\right. \\
&\left.\left.\left.\times\left|\cos \left(\phi_{0 l} / 2\right)\right| \sin (\alpha / 2)\right) \sin \theta_{l} \cot \left(\theta_{0 l}\right)\right] \hat{\boldsymbol{\theta}}_{l}\right\}
\end{aligned}
$$

where $z_{l}$ is the curvilinear coordinate along the rim. A local coordinate system $\left(O_{l}, \hat{\boldsymbol{x}}_{l}, \hat{\boldsymbol{y}}_{l}, \hat{\boldsymbol{z}}_{l}\right)$ is defined with $O_{l}\left(\boldsymbol{r}^{c}\right)$ on the rim, $\hat{\boldsymbol{z}}_{l}$ tangent to the rim, and $\hat{\boldsymbol{x}}_{l}$ orthogonal to $\hat{\boldsymbol{z}}_{l}$ in the plane tangent to the reflector surface at $O_{l} . E_{z_{l}}^{\mathrm{f}}$ and $H_{z_{l}}^{\mathrm{f}}$ are the $z_{l}$ components of the incident (feed produced) electric and magnetic fields, respectively, in this local coordinate system. The local spherical angular coordinates of the observation direction are denoted $\left(\theta_{l}, \phi_{l}\right)$ and those of an incident (locally) plane wave are $\left(\theta_{0 l}, \phi_{0 l}\right)$. Also, in (4),

$$
\alpha=\cos ^{-1}\left(\frac{\sin \theta_{l}}{\sin \theta_{0 l}} \cos \phi_{l}\right), 0 \leq \phi_{l}<2 \pi
$$

In general, full characterization of the far field pattern requires computation of $O\left(N_{\mathrm{a}}^{2}\right)$ of its samples. Here, $N_{\mathrm{a}}=k R_{\mathrm{a}}$ provides a measure of the antenna electrical size with $R_{\mathrm{a}}$ being the radius of the smallest sphere enclosing the whole antenna system. Complexity of the direct evaluation of (2) for $O\left(N_{\mathrm{a}}^{2}\right)$ observation directions is of $O\left(N_{\mathrm{a}}^{4}\right)$. This estimate is dominated by the cost of calculating the PO surface integral. However, the complexity of directly computing the PTD contour integral is of $O\left(N_{\mathrm{a}}^{3}\right)$ and, therefore, must also be addressed. Our goal is to reduce this overall computational cost to $O\left(N_{\mathrm{a}}^{2} \log N_{\mathrm{a}}\right)$, which is comparable to that of the FFT-based techniques that are used for planar apertures.

\section{Generalized MLPO ALGORIthm}

The generalized PO-PTD based numerical scheme is developed by extending the original MLPO approach. In a pre-processing phase, the reflector surface is hierarchically subdivided into subdomains: surface subdomains (also called "patches") at level $L$ are denoted by $S_{n}^{L}$, with $n=1, \ldots, N_{\mathrm{s}}^{L}$. Also, we denote $\overline{\boldsymbol{r}}_{n}^{L}$ and $R_{n}^{L}$ the center and the radius of the smallest sphere circumscribing patch $S_{n}^{L}$, while $R^{L}=$ $\max _{n}\left\{R_{n}^{L}\right\}$. If a binary subdivision scheme is used along each

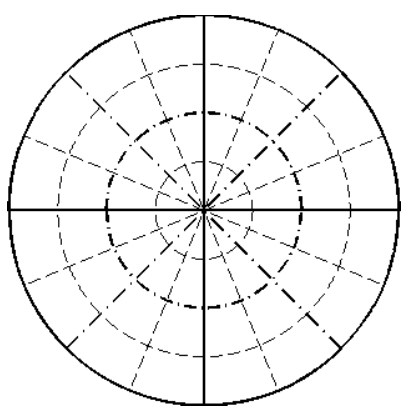

Fig. 1. Reflector surface decomposition along polar coordinates in the projection plane.

of the two coordinates spanning the surface, a parent patch of level $L$ is subdivided into four patches of level $L+1$, called its "children". An example of a binary subdivision in polar coordinates of the reflector projection plane is shown in Fig. 1. The subdivision process is stopped at level $L=M$ when $R^{M}$ is of the order of the wavelength.

The multilevel algorithm starts with the PO surface integration over each of the elemental patches of level $M$. These elemental subpatterns involve both the surface integrals of the equivalent currents (function $\boldsymbol{A}\left(\hat{\boldsymbol{r}}, \boldsymbol{r}^{s}\right)$ defined in (3)) and the line integrals of ILDC contributions for patches situated along the rim $C$, as defined in (4). This integration phase is thus performed for each patch $\left(n=1, \ldots, N^{M}\right)$ in its self-centered coordinate system (i.e., with the origin at point $\overline{\boldsymbol{r}}_{n}^{M}$ for patch $S_{n}^{M}$ ), yielding:

$$
\begin{aligned}
& \overline{\boldsymbol{U}}_{n}^{M}(\theta, \phi)=e^{-j k \hat{\boldsymbol{r}} \cdot \overline{\boldsymbol{r}}_{n}^{M}} \times \\
& \quad\left[\int_{S_{n}^{M}} \boldsymbol{A}\left(\hat{\boldsymbol{r}}, \boldsymbol{r}^{s}\right) e^{j k \hat{\boldsymbol{r}} \cdot \boldsymbol{r}^{s}} d s+\int_{S_{n}^{M} \cap C} \boldsymbol{D}\left(\hat{\boldsymbol{r}}, \boldsymbol{r}^{c}\right) e^{j k \hat{\boldsymbol{r}} \cdot \boldsymbol{r}^{c}} d z_{l}\right]
\end{aligned}
$$

Due to the size of these patches $\left(k R^{M}\right.$ is of $\left.O(1)\right)$, these radiation patterns are fully described by sampling the directions of observation very coarsely, according to Property 3 in [12]. The integrals are thus evaluated for a very sparse grid comprising $O(1)$, i.e., a small fixed number of directions. The number of quadrature points needed for surface and line integrals over each level $M$ patch is also of $O(1)$. The computational complexity of evaluating the integrals for each level $M$ patch is then of $O(1)$, and the total computational complexity of evaluating the surface and line integrals via (5) for all level $M$ patches is of $O\left(N_{\mathrm{r}}^{2}\right)$, where $N_{\mathrm{r}}=k R_{\mathrm{r}}$ denotes the electrical size of the reflector. Here, $R_{\mathrm{r}}$ denotes the radius of the smallest sphere circumscribing the whole reflector surface. It is noteworthy that the additional cost due to the evaluation of the PTD line integrals scales as $O\left(N_{\mathrm{r}}\right)$, and is expected to be quite small compared to that of the PO surface integrals.

The remainder of the algorithm involves multilevel aggregation of subdomain radiation patterns [11]. At each level from $M$ to 1 during the aggregation phase, the "children" patterns must be interpolated prior to aggregation, due to the need to increase the grid density with increasing subdomain size from level $L$ to level $L-1$. Thereafter they must be expressed in their "parent" patch coordinate system: the coordinate systems 
$\mathcal{S}_{n}^{L}$ with their origins at the $\overline{\boldsymbol{r}}_{n}^{L}$ points are translated to the coordinate system $\mathcal{S}_{m}^{L-1}$ with origin $\bar{r}_{m}^{L-1}$. Such translations are performed through phase changes in far field patterns. The obtained patterns can be summed and the resulting pattern is amenable to interpolation at the next aggregation step.

The computational complexity of such multilevel interpolation and aggregation process has been shown in [11] to be of $O\left(N_{\mathrm{r}}^{2} \log N_{\mathrm{r}}\right)$. Assuming that $N_{\mathrm{r}}$ is comparable with $N_{\mathrm{a}}$, the computational cost of this process is expected to asymptotically dominate the total cost of the radiation pattern evaluation for electrically very large antennas. On the other hand, for moderately sized reflectors, the level $M$ integration time tends to be the dominant computational burden, due to a large constant hidden in its $O\left(N_{\mathrm{r}}^{2}\right)$ complexity estimate.

If an antenna system comprises multiple radiating components such as the main and sub-reflectors, illuminated by single or multiple feed elements, the minimum sufficient sampling rates of individual radiation patterns can be applied to each object in its self-centered coordinate system. Interpolation and origin translations are then used to aggregate partial patterns into the global one, in the same way as for reflector subdomains. The density of angular grids is increased proportionally to the ratio of electrical sizes of individual components, leading to a final interpolation and aggregation step similar to the two-level Fast Physical Optics algorithm [12].

In the case of an offset single reflector antenna system presented in Fig. 2, the reflector and the feed can be considered as radiating objects with electrical sizes $N_{\mathrm{r}}$ and $N_{\mathrm{f}}$, and radiation patterns $\boldsymbol{U}_{\mathrm{r}}$ and $\boldsymbol{U}_{\mathrm{f}}$, respectively. Here, $\boldsymbol{U}_{\mathrm{r}}$ and $\boldsymbol{U}_{\mathrm{f}}$ are assumed to be computed in coordinate systems $\mathcal{S}_{\mathrm{r}}$ and $\mathcal{S}_{\mathrm{f}}$ with their origins at the centers of the smallest spheres circumscribing the reflector and the feed, respectively. Both the reflector and the feed patterns must be interpolated to the sampling rate associated with the radius $R_{\mathrm{a}}$ of the smallest enclosing sphere of the whole antenna. These interpolated patterns can then be translated to the global coordinate system with the origin at center of the antenna. Finally, the reflector and feed patterns can be summed to obtain the pattern $\boldsymbol{U}_{\mathrm{a}}$ of the whole antenna system. This final aggregation of the antenna pattern components is characterized by the complexity of $O\left(N_{\mathrm{a}}^{2}\right)$.

\section{NUMERICAL RESUlTS}

We apply the generalized MLPO algorithm to a single offset parabolic reflector antenna system used in the GRASP9 Technical Description document as an illustrative example of the computation of wide angle patterns (see [5], p. 241). The antenna configuration is defined by the following initial data (cf Fig. 2): parabolic reflector with diameter $D_{\mathrm{r}}=40 \lambda$, magnification factor $F / D_{\mathrm{r}}=0.8$, height of the reflector "center" with respect to the paraboloid axis $h=30 \lambda$, half angle subtended by the reflector from the source point at the reflector focus: $\theta=29.1^{\circ}$. A Gaussian feed is taken as the primary source, with its radiated fields computed by complex source point formulas (see [5], p. 99) with the complex shift parameter $b$ equal to $1.66 \lambda(12 \mathrm{~dB}$ taper at the edge of the reflector).

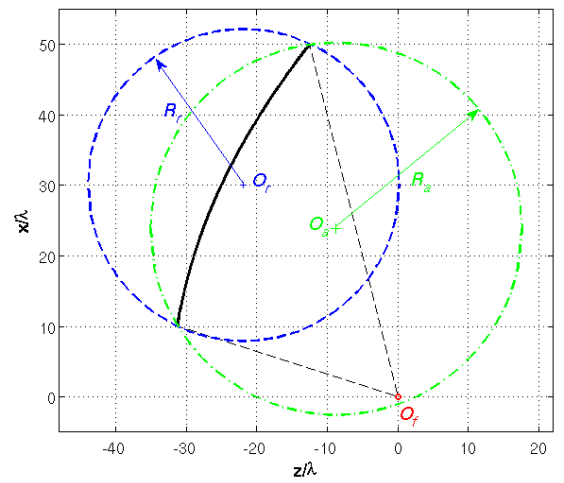

Fig. 2. Vertical cross section of the offset single reflector antenna system and spheres circumscribing the radiating components and the whole system. $\left(O_{\gamma}, R_{\gamma}\right)$ are the center and radius of the smallest sphrere circumscribing the feed $(\gamma=\mathrm{f})$, the reflector $(\gamma=\mathrm{r})$ and the whole antenna $(\gamma=\mathrm{a})$, respectively.

From these data, the MLPO code computes the radii of the spheres circumscribing the reflector and the whole antenna system, respectively. Using notations previously introduced for the reflector, the antenna system, and the feed, we obtain for the case under study: $R_{\mathrm{r}}=22.09 \lambda$ and $R_{\mathrm{a}}=25.98 \lambda$ (cf Fig. 2). $R_{\mathrm{f}}$ is taken equal to $b$. The number of decomposition levels $M$ for the multilevel computation of the reflector pattern is then computed so as to obtain sufficiently small patches at level $M$. For the above case, the computed value for $M$ is 5 , leading to $R^{M}=1.37 \lambda$ for the maximum radius of the level $M$ patch circumscribing spheres. A Gauss quadrature with 8 integrand points along each projected variable describing the reflector surface is used for surface integration over level $M$ patches. The PTD integral is performed with 8 integrand points along the reflector rim for each edge patch at level $M$.

Figure 3 presents the full 3D co- and crosspolarised patterns of the antenna system, including the PTD integral contribution and the feed spillover. These patterns were computed by our MLPO code in 253 seconds on a single processor, and comprise 2945 cuts with constant $\phi$ value, and $1473 \theta$ values in each of these cuts. To compute the same patterns with approximately the same accuracy $(1 \mathrm{~dB}$ down to $-80 \mathrm{~dB}$ on amplitude patterns) GRASP9 requires about $30 \mathrm{~min}$ on the same single processor machine.

Figure 4 illustrates the accuracy of the MLPO results by comparison with the patterns obtained with our home made direct PO code, and with GRASP9 reference results obtained with a large number of integration points on the reflector surface. The cut shown on this figure is in the plane of symmetry of the patterns ( $\mathrm{xOz}$ plane: $\phi=0, \pi)$, where the requirements on the surface and edge integrals are particularly high to reach the prescribed accuracy of $1 \mathrm{~dB}$ down to $-80 \mathrm{~dB}$.

For the considered antenna, the shadow boundaries in the symmetry plane occur in the $\theta_{t}=104.2^{\circ}$ (top of the reflector) and $\theta_{b}=162.2^{\circ}$ (bottom of the reflector) directions, shown on Fig. 2 (black dashed lines). The feed radiation is shadowed in the $\left[\theta_{t}, \theta_{b}\right]$ interval of $\theta$ values. As a consequence, the pattern shown in Fig. 4 exhibits an increase of the back radiation around these directions. It should be noted that the respective fields computed by surface integration and 


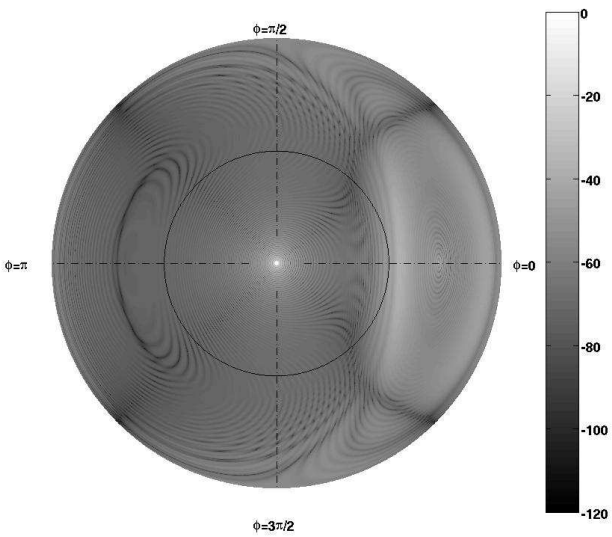

(a) Copolarized pattern.

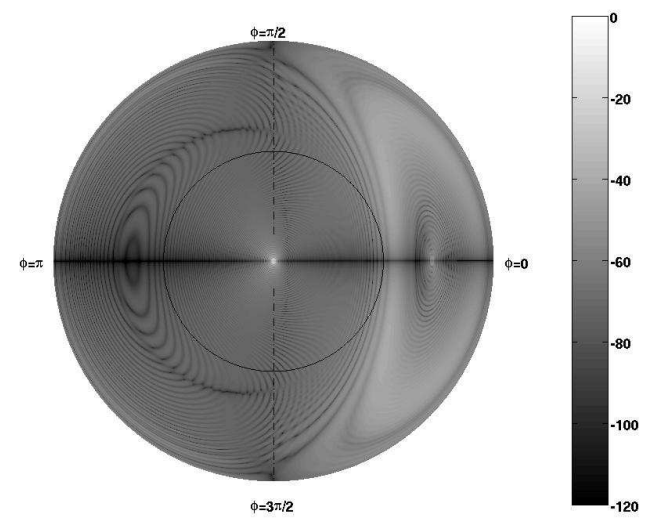

(b) Crosspolarized pattern.

Fig. 3. 3D patterns computed with MLPO, including PTD contribution. $\theta$ is varying from 0 to $\pi$ along the radial coordinate. The black circle is for $\theta=\pi / 2$, i.e. the boundary between the front and rear patterns.

edge integration in PO-PTD simulations are quite different from the fields obtained in GO-GTD based simulations from specular reflection of space rays and from edge diffracted rays, respectively. In the latter type of simulation, the rays originating from the feed are blocked by the reflector, yielding a GO field discontinuity along the shadow boundary. On the contrary, the far field obtained by PO surface integration is not null in the angular region "shadowed" by the reflector. Combination of the field obtained by PO integration with the direct radiation from the feed yields the shape of the "shadow" pattern, except in angular regions where the PTD contribution is dominant. For instance, the peak which is observed for $\theta \sim 130^{\circ}$ in Fig. 4, corresponds both to the direction of maximum radiation of the feed and of equiphase combination of contributions from elliptical slices of the reflector surface parallel to its edge; in GO-GTD simulations, it appears as a caustic for GTD rays, while GO rays are blocked in that direction [5]. The influence of the PTD integral is illustrated in Fig. 5 on two cuts of full 3D patterns obtained with and without the PTD integral contribution. This influence is visible, both on the copolarized pattern in the symmetry plane $(\phi=0)$ and on the crosspolarized pattern in the $\phi=\pi / 4$ plane. The most critical region with respect to the integration accuracy is in the symmetry plane, in the interval of $\theta$ values ranging between $-140^{\circ}$ and $-90^{\circ}$, i.e., in the back radiation pattern not only of the reflector but also of the feed (cf Fig. 2). The other region where the PTD contribution is clearly dominant on this pattern, approximately between $40^{\circ}$ and $70^{\circ}$, shows the importance of taking into account the PTD fields in far sidelobe regions, even in the front radiation pattern.

Finally, we illustrate the robustness of the MLPO algorithm, by increasing the size of the problem, showing that parameters tuned for a small wide angle pattern problem lead to prescribed accuracy for large sized problems. Increasing the problem size leads to an increased number of decomposition levels, which is determined by the code itself. Oversampling values and numbers of quadrature points are kept the same for all problem sizes, as they only depend on the patch size at the highest level of decomposition. To validate this approach, large antenna problems are constructed by scaling the previously described one, considered as the "scale 1" configuration. Scaling by a factor $q$ is performed by multiplying the frequency by $q$.

Applying the MLPO algorithm to the scale 4 configuration, with the scale 1 oversampling values and numbers of quadrature points, leads to an observed accuracy of $1 \mathrm{~dB}$ down to $-90 \mathrm{~dB}$ on the amplitude 3D patterns. Table I shows representative values for MLPO and direct PO computation times in seconds, obtained for the scale 1 and scale 4 problems with a single Intel Xeon X5460@3.16GHz processor on a multiuser server for pattern computations without PTD integral ("only PO" column) and with PTD integral ("with PTD" column). The increase in the computation time when accounting for the PTD contribution is presented in the last column. Computation times are subject to variations from one run to another, but these values are "representative" in the sense that they were obtained repeatedly with less than $1 \%$ or a few seconds of error. The direct PO computation times for sufficiently sampled wide angle patterns of the scale 4 antenna system were obtained by multiplying by a proper factor the computation times measured when computing undersampled subsets of the full patterns (with no adaptive quadrature rule being used, CPU times are essentially proportional to the number of directions of the computed patterns for large

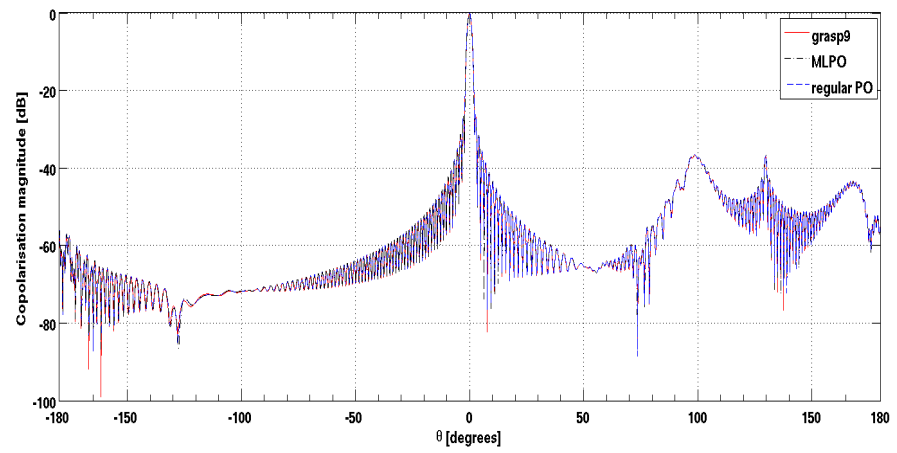

Fig. 4. Co-polarized pattern cut in the symmetry plane: comparison between GRASP9, MLPO, and direct PO results. 


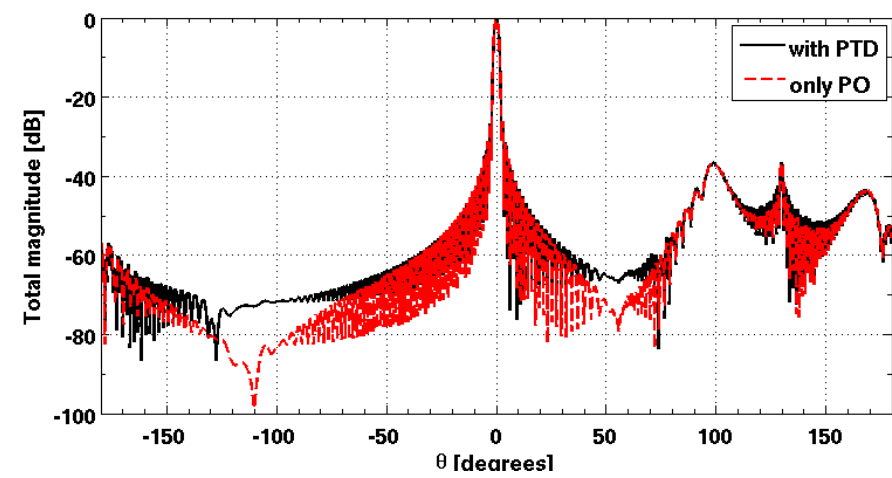

(a) Copolarized pattern in the symmetry plane.

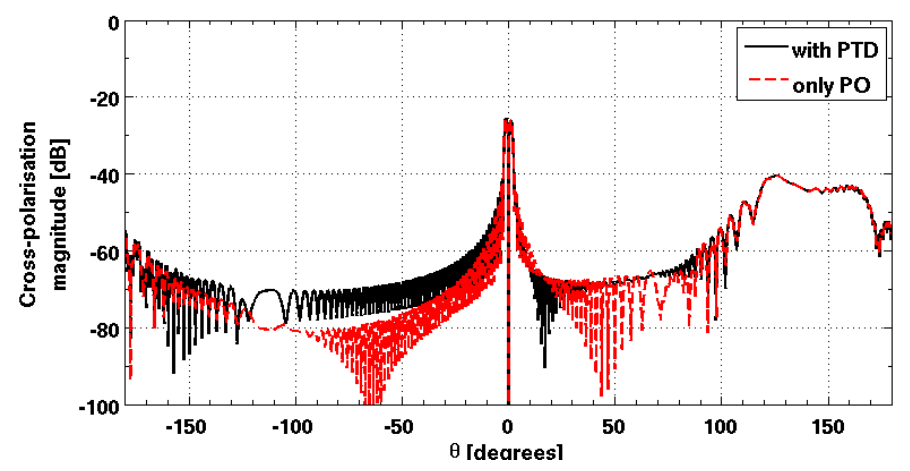

(b) Crosspolarized pattern in the $\phi=\pi / 4$ plane.

Fig. 5. Comparison of the patterns computed by the PO surface integral only, and by the PO + PTD integrals. The fields radiated by the feed are added in both cases.

antenna systems). The "ratio" row in the Table is obtained by dividing computation times of scales 4 and 1: in the case of computations involving only surface integrals, this ratio is expected to be close to 16 for the MLPO, and of the order of 256 for the direct PO. The measured values are clearly in good agreement with these predictions.

The data of Table I support the claim that the PTD contribution must also be computed by a multilevel algorithm, in order to keep its computation time negligible with respect to the MLPO surface integral computation. The ratio of computation times devoted to the PTD integral should theoretically vary as $N_{\mathrm{r}}$ with MLPO and $N_{\mathrm{r}}^{3}$ with regular PO (leading to ratios of 4 and 64 respectively). The orders of magnitude of the experimental data presented here (4.3 and 92., respectively) are coherent with these predictions. Computation of the PTD contribution by the direct numerical integration at scales 1 and 4 requires minutes and hours, respectively, in contrast to the computation times of a few seconds (less than $20 \mathrm{~s}$ at scale 4) observed with the MLPO.

\section{Conclusion}

The MLPO algorithm augmented with the PTD integral has been developed and applied to a single reflector antenna. Furthermore, the PO and PTD contributions of the reflector have been combined with the feed radiation to obtain the total far field pattern of the antenna over the whole angular range. The algorithm accuracy and computational efficiency have been tested on the case of full 3D pattern computations
TABLE I

COMPUTATION TIMES IN SECONDS OF THE MLPO AND DIRECT PO ALGORITHMS, FOR THE SCALE 1 AND SCALE 4 PROBLEMS, WITH AND WITHOUT THE PTD LINE INTEGRAL

MLPO algorithm CPU times (s)

\begin{tabular}{|c|c|c|c|}
\hline & only PO & with PTD & PTD cost \\
\hline scale 1 & $1.537 \cdot 10^{2}$ & $1.572 \cdot 10^{2}$ & 3.5 \\
\hline scale 4 & $2.270 \cdot 10^{3}$ & $2.285 \cdot 10^{3}$ & $1.5 \cdot 10^{1}$ \\
\hline ratio 4/1 & 14.8 & 14.5 & 4.3 \\
\hline
\end{tabular}

direct PO algorithm CPU times (s)

\begin{tabular}{|c|c|c|c|}
\hline & only PO & with PTD & PTD cost \\
\hline scale 1 & $2.20 \cdot 10^{3}$ & $2.32 \cdot 10^{3}$ & $1.2 \cdot 10^{2}$ \\
\hline scale 4 & $5.78 \cdot 10^{5}$ & $5.89 \cdot 10^{5}$ & $1.1 \cdot 10^{4}$ \\
\hline ratio 4/1 & 263. & 254. & 92. \\
\hline
\end{tabular}

by comparison to reference results. It has been shown to easily satisfy the accuracy required for wide angle patterns in the demanding case of a single offset reflector antenna.

\section{ACKNOWLEDGMENT}

The authors would like to thank Dr. Erik Jorgensen of TICRA for providing reference results obtained with the GRASP9 software.

\section{REFERENCES}

[1] Y. Rahmat-Samii, "Reflector antennas," in Antenna Handbook, Y.-T. Lo and S.-W. Lee, Eds. New York: Van Nostrand Reinhold, 1993, vol. II, pp. 15:1-124.

[2] R. Shore and A. Yaghjian, "Application of Incremental Length Diffraction Coefficients to calculate the pattern effects of the rim and surface cracks of a reflector antenna," IEEE Trans. Antennas Propagat., vol. 41, pp. 1-11, Jan. 1993.

[3] - "Correction to application of Incremental Length Diffraction Coefficients to calculate the pattern effects of the rim and surface cracks of a reflector antenna," IEEE Trans. Antennas Propagat., vol. 45, p. 917, May 1997.

[4] A. Miura and Y. Rahmat-Samii, "Spaceborne mesh reflector antennas with complex weaves: Extended PO/periodic-MoM analysis," IEEE Trans. Antennas Propagat., vol. 55, pp. 1022-1029, Apr. 2007.

[5] Technical Description of GRASP9, K. Pontoppidan, ed., TICRA, 2005, www.ticra.com.

[6] V. Galindo-Israel, T. Veruttipong, S. Rengarajan, and W. Imbriale, "Inflection point caustic problems and solutions for high-gain dualshaped reflectors," IEEE Trans. Antennas Propagat., pp. 202 -211, Feb. 1990.

[7] D.-W. Duan and Y. Rahmat-Samii, "A generalized diffraction synthesis technique for high performance reflector antennas," IEEE Trans. Antennas Propagat., vol. 43, pp. 27-40, Jan. 1995.

[8] Y. Z. Umul, "Modified theory of physical optics solution of impedance half plane," IEEE Trans. Antennas Propagat., vol. 54, pp. 2048-2053, July 2006.

[9] T. Shijo, L. Rodriguez, and M. Ando, "The modified surface-normal vectors in the physical optics," IEEE Trans. Antennas Propagat., vol. 56, pp. 3714-3722, Dec. 2008.

[10] R. Hoferer and Y. Rahmat-Samii, "Subreflector shaping for antenna distortion compensation: an efficient Fourier-Jacobi expansion with GO/PO analysis," IEEE Trans. Antennas Propagat., vol. 50, pp. 1676 1687, Dec. 2002.

[11] A. Boag and C. Letrou, "Multilevel Fast Physical Optics algorithm for radiation from non-planar apertures," IEEE Trans. Antennas Propagat., vol. 53, pp. 2064-2072, June 2005.

[12] _ - "Fast Physical Optics algorithm for lens and reflector antennas," IEEE Trans. Antennas Propagat., vol. 51, pp. 1063-1068, May 2003. 